\title{
Wearable cardioverter defibrillators: dead on arrival or chance of survival?
}

\author{
Pooja S. Jagadish ${ }^{1}$, Rami N. Khouzam ${ }^{2}$ \\ ${ }^{1}$ Department of Internal Medicine, University of Tennessee Health Science Center, Memphis, TN, USA; ${ }^{2}$ Division of Cardiovascular Diseases, \\ Department of Internal Medicine, University of Tennessee Health Science Center, Memphis, TN, USA \\ Correspondence to: Pooja S. Jagadish, MD. Department of Internal Medicine, University of Tennessee Health Science Center, 956 Court Ave., Suite \\ H314, Memphis, TN 38163, USA. Email: jagadish@uthsc.edu.
}

Submitted Jun 11, 2019. Accepted for publication Jun 28, 2019.

doi: $10.21037 / \mathrm{atm} .2019 .07 .04$

View this article at: http://dx.doi.org/10.21037/atm.2019.07.04

Sudden cardiac death affects over 300,000 individuals annually in the United States (1-4), and it comprises $40-50 \%$ of total cardiovascular disease-related deaths (3). The wearable cardioverter defibrillator (WCD) gained FDA approval in 2002 (5) and is a guideline-directed therapy intended to reduce the risk of sudden cardiac death from death from ventricular tachyarrhythmias, including ventricular fibrillation (VF) or ventricular tachycardia (VT) $(2,6)$. However, it is well-documented that ideal use of the WCD (trade name LifeVest ${ }^{\circledR}$ ) is constrained by size and weight of the device as well as development of a rash (7-9). The recent groundbreaking research released by the VEST Trial investigators has cast further doubt on the validity of the device by failing to demonstrate a significantly lower rate of death from tachyarrhythmias among WCD users (10). Also considering that premature discontinuation rates are as high as $30 \%$ (11), this begs the question: Is the WCD dead on arrival, or does it have a chance of survival with some modifications?

The LifeVest $(2,7,12)$ comes in a single size for both men and women, weighing $1.5 \mathrm{~kg}$ (13). It is comprised of a rechargeable external battery pack and cardiac monitor as well as a fabric vest-like garment with electrodeembedded pads that are worn directly touching the skin of the back. The front of the vest is an adjustable band worn horizontally below the breasts that are connected to the back by two vertical straps worn over the lateral aspects of the breasts. The device continuously monitors a patient's cardiac rhythm (5) and can defibrillate a patient up to five times at 150 Joules each when a ventricular tachyarrhythmia is detected (7). If conscious patients hear the device beeping, indicating an imminent shock, they may abort the sequence by pushing two buttons simultaneously $(3,7)$.

The most pressing issue is addressing the size and weight of the device. In an era when batteries are becoming smaller and obesity rates are climbing, it would make sense to modify the device to better suit its target audience. Obese patients can have trouble fitting into the device without appropriate fitting (2). Furthermore, patients with excess fat around the chest are more likely to have electrocardiographic artifact compared to patients of a normal or even overweight body mass index (12). This leads to inappropriate alarming, which may wake patients up from sleep or cause public embarrassment, contributing to premature discontinuation $(14,15)$. In fact, one study by Lackermair et al. reported that $48 \%$ of the 109 studied patients reported sleep disturbance with increased alarms contributing to higher levels of anxiety about shock therapy $(\mathrm{P}=0.03)(15)$. While studies have suggested no decreased WCD use in obese patients (12), much of the overall premature discontinuation of the WCD is related to discomfort (8). Thus, it is imperative to address the size of the device.

Next, rash is another reason cited for premature discontinuation $(7,8)$. Feldman et al. noted that a full $6 \%$ of patients $(\mathrm{n}=17)$ developed a hypersensitivity reaction to the device (8). Erath et al. documented in two patients a proven nickel hypersensitivity that was refractory to topical therapy; this allergy forced discontinuation of the WCD (14). The possibility of simple atopic dermatitis, development of folliculitis, or miliaria from sweat trapped between the device's pads and the skin can decrease comfort. While 
the device manual shows how to wash the fabric portion of the device, other components cannot contact water and are harder to clean. This hampers the optimal cleanliness of the WCD. Addressing the device's breathability and its propensity of causing a rash can improve patient comfort.

There are many ways to address the design concerns of the WCD. First, instead of using a bulky monitor/battery pack, the monitor can be downsized to either an app on the patient's smartphone or to a smartphone-sized wirelessand Bluetooth-enabled device that is kept discreetly in a pocket or handbag. This device would then port data to the company's servers. Next, the battery pack should be made more compact. While a battery the size of an implantable cardioverter defibrillator is unlikely, given that the electric current must penetrate flesh to get to the heart, it can likely at least be made wireless. To prevent users from forgetting to carry it, it can have a unique alarm that sounds when a user is out of range. Ideally, it would also be small enough to carry in a pocket or a handbag. With the two previous components being wireless, the electrodes and pads could be single-use and flexible with pores to make them more breathable. They could be affixed on the skin in a similar location to either automated external defibrillator pads or on the patient's back, as with the current WCDs. For easier application to the back, a tool may need to be provided to help a patient reach the correct location. These three changes would also reduce the risk of nickel hypersensitivity and rash development, and hypoallergenic adhesive can further reduce adverse effects. This updated device would be more easily worn under clothes and applied to bulkier areas of the body with a decreased risk of electrocardiographic noise.

Currently, WCDs only target ventricular tachyarrhythmias, and they have no role in non-VT/VF rhythms, such as bradyarrhythmias (6). Bradyarrhythmias are a documented cause of death among patients wearing WCDs (14). With the device's current battery capacity, transcutaneous pacing could be an added function to bridge patients until they arrive at an emergency department for further care. In fact, the device may be totally overhauled to become an in-hospital pacing device.

It remains to be determined the effect of the VEST trial on the role of the WCD in current guidelines. Although the VEST trial demonstrates decreased allcause mortality, WCD therapy failed to reduce the primary endpoint of arrhythmic death in the first 90 days postmyocardial infarction (10). As with previous studies, there was significant premature discontinuation that increased with time (10). The major limitations of the VEST trial include its intention-to-treat analysis, wherein those who discontinued the device are still considered part of the WCD group; a full $75 \%$ of deaths in the treatment group were among device-non-adherent patients (10). This fosters the argument that with device modification and increased adherence to therapy, perhaps results may have been different.

As it stands, the WCD has Class IIb standing for primary prevention in recent post-myocardial infarction patients with ischemic heart disease and a left ventricular ejection fraction (LVEF) below $40 \%$ as well as for secondary prevention in newly diagnosed nonischemic cardiomyopathy patients with class II-III heart failure and an LVEF below $35 \%$. There are device modifications that can improve function and use of the device and perhaps even expand its market and functionality, warranting reconsideration of its validity. Without changes, however, the LifeVest may just be dead on arrival.

\section{Acknowledgments}

None.

\section{Footnote}

Conflicts of Interest: The authors have no conflicts of interest to declare.

Ethical Statement: The authors are accountable for all aspects of the work in ensuring that questions related to the accuracy or integrity of any part of the work are appropriately investigated and resolved.

\section{References}

1. Benjamin EJ, Virani SS, Callaway CW, et al. Heart Disease and Stroke Statistics-2018 Update: A Report From the American Heart Association. Circulation 2018;137:e67-492.

2. Piccini JP Sr, Allen LA, Kudenchuk PJ, et al. Wearable Cardioverter-Defibrillator Therapy for the Prevention of Sudden Cardiac Death: A Science Advisory From the American Heart Association. Circulation 2016;133:1715-27.

3. Agarwal M, Narcisse D, Khouzam N, et al. Wearable Cardioverter Defibrillator "The Lifevest": Device Design, Limitations, and Areas of Improvement. Curr Probl Cardiol 2018;43:45-55. 
4. Kuriachan VP, Sumner GL, Mitchell LB. Sudden cardiac death. Curr Probl Cardiol 2015;40:133-200.

5. Sharma PS, Bordachar P, Ellenbogen KA. Indications and use of the wearable cardiac defibrillator. Eur Heart J 2017;38:258-67.

6. Al-Khatib SM, Stevenson WG, Ackerman MJ, et al. 2017 AHA/ACC/HRS Guideline for Management of Patients With Ventricular Arrhythmias and the Prevention of Sudden Cardiac Death: A Report of the American College of Cardiology/American Heart Association Task Force on Clinical Practice Guidelines and the Heart Rhythm Society. J Am Coll Cardiol 2018;72:e91-220.

7. Chung MK. The role of the wearable cardioverter defibrillator in clinical practice. Cardiol Clin 2014;32:253-70.

8. Feldman AM, Klein H, Tchou P, et al. Use of a wearable defibrillator in terminating tachyarrhythmias in patients at high risk for sudden death: results of the WEARIT/ BIROAD. Pacing Clin Electrophysiol 2004;27:4-9.

9. Jagadish PS, Aziz M, Chinta V, et al. Misunderstood or Mistrusted? The Under-Utilization of the Wearable Cardioverter Defibrillator in Clinical Practice. Curr Probl Cardiol 2018. [Epub ahead of print].

Cite this article as: Jagadish PS, Khouzam RN. Wearable cardioverter defibrillators: dead on arrival or chance of survival? Ann Transl Med 2019;7(17):403. doi: 10.21037/atm.2019.07.04
10. Olgin JE, Pletcher MJ, Vittinghoff E, et al. Wearable Cardioverter-Defibrillator after Myocardial Infarction. N Engl J Med 2018;379:1205-15.

11. Chung MK, Szymkiewicz SJ, Shao M, et al. Aggregate national experience with the wearable cardioverterdefibrillator: event rates, compliance, and survival. J Am Coll Cardiol 2010;56:194-203.

12. Wan C, Szymkiewicz SJ, Klein HU. The impact of body mass index on the wearable cardioverter defibrillator shock efficacy and patient wear time. Am Heart J 2017;186:111-7.

13. Francis J, Reek S. Wearable cardioverter defibrillator: a life vest till the life boat (ICD) arrives. Indian Heart J 2014;66:68-72.

14. Erath JW, Vamos M, Sirat AS, et al. The wearable cardioverter-defibrillator in a real-world clinical setting: experience in 102 consecutive patients. Clin Res Cardiol 2017;106:300-6.

15. Lackermair K, Schuhmann CG, Kubieniec M, et al. Impairment of Quality of Life among Patients with Wearable Cardioverter Defibrillator Therapy (LifeVest $\left.{ }^{\circledR}\right)$ : A Preliminary Study. Biomed Res Int. 2018;2018:6028494. 